It is clear that trainees are struggling to balance multiple commitments and that the SLT is being viewed by some as another 'tick box' exercise. Hopefully some trainees have found it a useful tool for developing vital skills.

\section{IMPROVING PATIENTSAFETY CULTURE IN THE PRIMARY CARE SETTING}

${ }^{1}$ A Hollis*, 'Leo Feinberg, ${ }^{2}$ Will Murdoch. 'Sandwell and West Birmingham NHS Trust, Birmingham, West Midlands; ${ }^{2}$ The Modality Partnership, Birmingham, West Midlands

10.1136/leader-2019-FMLM.88

Aims To improve engagement with the incident reporting process and to encourage staff to raise issues and create a proactive culture of quality improvement.

Methods All staff members were invited to take part in the GP Safety Climate Questionnaire pre and post intervention to assess self-reported feelings towards patient safety at the practice. We then developed a novel incident reporting tool which was introduced in collaboration with an adapted 'Take off, cruising and landing' daily safety huddle. The incident reporting tool was designed to be as simple as possible, easy to access and quick to complete.

Results Prior to the new tool and adapted safety huddle we had 6 significant event forms completed within the practice in the preceding three months. Following implementation, we had a total of 191 incident forms over a ten-week period (which aimed to capture all issues from low level to significant events). These issues highlighted several process issues within our GP practice namely issues with appointments, inter-practice communication and prescriptions. The safety huddle also allowed issues to handed over from one day to the next formally. GP safety climate questionnaire scores also showed a substantial improvement with below average scores (compared with other practices) pre implementation improving to above average scores post.

Conclusions This project demonstrates that a relatively simple intervention can have effect significant positive cultural change in an organisation over a small period of time. Through this project we were able to generate a wealth of information that we could use to target areas of improvement. By giving frontline staff a mechanism to record issues it is possible to develop a positive culture of grass roots change. Incident reporting can act as a vehicle not only to improve patient safety but more broadly to generate ongoing ideas for quality improvement within an organisation.

\section{IMPROVING CARDIOLOGY REFERRAL PROCESS AT ROYAL SURREY COUNTY HOSPITAL}

P Bamania*, A Mellan, K Moosai, Z Zuberi. Royal Surrey County Hospital, Guildford, UK

\subsection{6/leader-2019-FMLM.89}

Background The Cardiology referral system at Royal Surrey County Hospital involved bleeping the registrar. It is often the junior doctors' job to make the referral, and occasionally not all information is at hand.

Aim To improve efficiency of inpatient Cardiology referral process by creating an easy-to-use system enabling effective timely referrals with relevant information.
Methods Questionnaires were distributed to junior doctors with five closed questions, rated strongly agree to strongly disagree. A proforma was designed and implemented with feedback from the Cardiology team. Improvements were measured objectively through questionnaires and verbal feedback.

Leadership This project began with identifying an area for improvement, reviewing and planning for standardised system. It involved working closely with the Cardiology team, through meetings and email correspondence. We also took into consideration suggestions from clinicians outside Cardiology. We evaluated referral systems in other specialities, which helped aid design.

Results Before implementation: 22 junior doctors responded. $50 \%$ agreed they knew how make to make a Cardiology referral, $50 \%$ disagreed. $68 \%$ disagreed that it is very easy to get a referral. 50\% agreed they included the correct information. $55 \%$ agreed they were often told to gather more information. $77 \%$, spent two hours or more trying to refer.

Two months after implementation: 21 junior doctors responded. $81 \%$ agreed they knew how make to make a Cardiology referral. $67 \%$ agreed that it is very easy to get a cardiology referral. 57\% agreed they always included the correct information. 24\% agreed they were asked to gather more information. All referrals were completed within an hour.

Conclusion The implementation of these changes highlights the key qualities needed in leadership and management good communication skills, being innovative as well as challenges one may face and being able to use feedback to guide positive change.

\section{YOUR'RE HIRED! DEVELOPING CLINICAL LEADERS OF TOMORROW USING THE GOVERNMENT APPRENTICESHIP LEVY}

${ }^{1}$ Bill Kawai-Calderhead, ${ }^{2}$ Roddy Christie. ${ }^{1}$ Royal Army Medical Corps, British Army; ${ }^{2}$ Always Consult Ltd, UK

\subsection{6/leader-2019-FMLM.90}

Aim Medical leadership is recognised as an essential facet of clinical practice. However there lacks standardised, sustainable training for postgraduate doctors in particular to support the transition from undergraduate medical student to Foundation Year 1 (FY1) doctor. This challenging transition creates significant anxiety amongst FY1s and clinician burnout is a national concern. Through the Government Apprenticeship Scheme NHS Trusts have access to a ring-fenced budget that can provide funded, vocationally based, nationally benchmarked leadership and management (LM) training which can support FY1s during this transition.

Methods In December 2017, through a partnership between the authors, South Tees Hospitals NHS Foundation Trust and Always Consult, a Registered Apprenticeship Training provider, the Foundation Leadership and Management (FLM) programme for FY1s was launched. FLM consists of 12 LMthemed modules which complement the FY1 clinical curriculum and leads to a nationally recognised qualification enabling membership of two leading international LM bodies. Participants are regularly surveyed for their self-rated preparedness for the LM challenges of FY1 practice and resilience through the Brief Resilience Score (BRS). 
Results Those on the FLM Apprenticeship showed greater selfrated preparedness for the LM challenges of practice when compared to those not on the Apprenticeship. Participants' first BRS in October 2018 was taken as a baseline and compared with their last BRS in January or March 2019. Increases in BRS were observed in over $70 \%$ of those on the FLM Apprenticeship compared to decreases in over $70 \%$ of those not on the Apprenticeship.

Conclusions LM programmes such as FLM can increase FY1s' self-rated LM preparedness and resilience. Apprenticeships offer an opportunity to establish locally deliverable LM programmes which holistically benefit staff in a resource constrained and challenging environment.

\section{SYSTEMS LEADERSHIP LESSONS FROM PLANNING FOR WINTER PRESSURES}

${ }^{1}$ Christopher J Miller, ${ }^{2}$ Rebecca Hall, ${ }^{3}$ Melanie McFeeters, ${ }^{4}$ Alyson Evans, ${ }^{4}$ Emma Orrock, ${ }^{5}$ Matt Day. ${ }^{1}$ University Hospitals of Leicester NHS Trust; ${ }^{2}$ Medical Centre Loughborough; ${ }^{3} \mathrm{NHS}$ England and Improvement - Midlands; ${ }^{4}$ East Midlands Clinical Senate; ${ }^{5}$ Public Health England East Midlands

\subsection{6/leader-2019-FMLM.91}

Aims Unprecedented winter pressures in 2017-18 led to cancellations of elective activity across the NHS and deteriorating population outcomes. East Midlands Clinical Senate undertook a review of the local planning processes to manage periods of increased demand

Methods A two-stage approach was adopted:

- Literature review of national guidance

- Interviews with four local organisations

Conclusion 25 papers were identified with guidance falling within 8 key themes: patient flow $(n=13)$, communication and collaboration (13), demand (13), system capacity (12), funding (6), planning (6), patient safety (3) and staff training (3)

The interviews revealed that despite similar system and operational challenges, clinical solutions are not being shared between commissioners and providers at scale

More collaborative systems leadership approaches from commissioners during winter 2018-19 resulted in improved outcomes across the system

The ambulance service is uniquely placed as a provider which interacts daily across local systems and with providers. This enables operational and clinical relationships to be developed and there was evidence of how a strategic approach to learning and responding to this insight had enabled better performance and outcomes following the winter of 2017-18

The importance of looking after workforce and creating the best possible conditions for staff to do their best for patients was highlighted

Recommendations Better sharing of data and practices across the region

Systems embrace and develop innovative ways of working to facilitate holistic care

Commissioners should consider acting more as facilitators (enable change to occur and issues to be tackled) rather than regulators (seeking assurances on what has already happened)
Develop better relationships with ambulance services to ensure practices are robust and standardised

Improvements in rotas and increased investment in staff (both in terms of staffing numbers and improved skillset of each staff member)

\section{TELEDERMATOLOGY: AN INNOVATIVE APPROACH TO MANAGE SKIN CONDITIONS IN PRIMARY CARE}

S Zuhairy. County Durham and Darlington Foundation Trust

10.1136/leader-2019-FMLM.92

Teledermatology is a newly growing service delivering dermatologic care, covering the entire field of dermatology, ranging from initial diagnosis to treatment and management. Teledermatology involves the use of medical photography to examine the skin of a patient together with the relevant history to diagnose dermatological conditions. Images are sent electronically to the dermatologist and in most cases receive a response within 48 hours. GP practices have started making diagnostic agreements with GP's with a special interest in Dermatology. Teledermatology gives the opportunity for the majority of patients to be treated by their own GP/Primary care, following a suitable management plan suggested by the GP with a special interest in Dermatology, but without having to attend hospital. By reducing the number of dermatology referrals to secondary care, Teledermatology can also help address the demand for dermatology care in hospitals. Teledermatology has shown to optimize resourses, provide quick and efficient care as well as reducing the number of referrals to secondary care. It is a service that should be achieved by all primary care physicians. Teledermatology has the potential to permanently change the way that doctors manage their workflow and provide patient care.

\section{$93 \quad$ INNOVATING IN CHILDREN'S URGENT CARE}

${ }^{1}$ Mathew Mathai, 1,2Reena Basu. 'Bradford Teaching Hospitals Foundation Trust, UK; ${ }^{2}$ Health Education UK

\subsection{6/leader-2019-FMLM.93}

Aim The number of emergency department attendances by children and young (CYP) is on the rise. However, 90\% of medical patients are discharged after initial assessment. The Ambulatory Care Experience (ACE) service is a new model for urgent care for CYP. The primary aim of the team is to provide child and family centred quality acute care at the right time and in the right place, ideally at home. The service provides an alternative to a hospital referral or admission for CYP.

\section{ACE Intervention}

1. Paediatric consultants take clinical responsibility from point of referral. Subsequently, CYP receive up to 5 days of home observation, delivered by a specially trained band 6 nurse.

2. Development of the service has required a system wide, shared ambulatory care vision. It was designed with families 